\title{
Detection of ctDNA in the plasma of patients with papillary thyroid carcinoma
}

\author{
HUIQIANG LI ${ }^{1 *}$, JIANGMAN ZHAO ${ }^{2,3^{*}}$, JIANHUA ZHANG ${ }^{1}$, CONGREN WANG $^{1}$, \\ MINGZHU LI ${ }^{1}$, SHOUXIN WU ${ }^{2,3}$, ZIJIAN SU ${ }^{1}$ and QUNXIONG PAN ${ }^{1}$ \\ ${ }^{1}$ Department of Oncology Surgery, Affiliated Quanzhou First Hospital of Fujian Medical University, Quanzhou, Fujian 362000; \\ ${ }^{2}$ Zhangjiang Center for Translational Medicine, Shanghai Biotecan Pharmaceuticals Co., Ltd., Shanghai 201203; \\ ${ }^{3}$ Department of Medicine, Shanghai Zhangjiang Institute of Medical Innovation, Shanghai 201204, P.R. China
}

Received February 15, 2019; Accepted August 2, 2019

DOI: $10.3892 /$ etm.2019.7997

\begin{abstract}
A total of $15-30 \%$ of thyroid nodules that are evaluated by fine-needle aspiration are not clearly determined to be benign or malignant. Gene mutation analysis is recommended for the evaluation of thyroid nodules using clinical guidelines. The detection of circulating tumor DNA (ctDNA) has the potential to aid in the screening, diagnosis and prediction of thyroid cancer prognosis, and can be used when tissues are difficult to obtain. In the present study, whole-exome sequencing (WES) was performed on tumors and matched normal tissues from 10 patients with papillary thyroid carcinoma (PTC) in Quanzhou, China. Hotspot mutations in tumor DNA and cell-free DNA were identified in the validation cohort, which included 59 patients with PTC. BRAF V600E occurred in five samples, and was the most frequent mutation observed. Variation allele frequency (VAF) of BRAF V600E detected by WES was positively correlated with VAF determined using digital PCR $\left(\mathrm{R}^{2}=0.9197 ; \mathrm{P}=0.0099\right)$. A number of novel mutated genes were identified, including zinc finger protein 717, pleckstrin homology like domain family A member 1, RBMX like 3, lysine methyltransferase $5 \mathrm{~A}$ and trichohyalin, along with the reported genes BRAF, NRAS and mucin 16, cell surface associated. Somatic mutated genes were enriched in the 'focal adhesion' pathway, as determined by Kyoto Encyclopedia of Genes and Genomes or Gene Ontology analysis. In the validation cohort, $44.07 \%$ of tumors were BRAF V600E-positive, and the sensitivity and
\end{abstract}

Correspondence to: Dr Qunxiong Pan or Dr Zijian Su, Department of Oncology Surgery, Affiliated Quanzhou First Hospital of Fujian Medical University, 248-252 East Street, Quanzhou, Fujian 362000, P.R. China

E-mail: 710262737@qq.com

E-mail: zijiansue@126.com

*Contributed equally

Key words: papillary thyroid carcinoma, whole-exome sequencing, circulating tumor DNA, liquid biopsy, focal adhesion specificity of BRAF V600E ctDNA were 61.54 and $90.91 \%$, respectively. BRAF V600E was associated with aggressive tumor factors, including lymph node metastasis $(\mathrm{P}=0.001)$ and advanced disease stage $(\mathrm{P}=0.009)$. The present study investigated the accuracy of ctDNA detection in patients with PTC, and provided evidence that ctDNA can be used as an evaluation of tumor DNA in thyroid nodules.

\section{Introduction}

Thyroid cancer (TC) is a common malignancy of the endocrine system. According to the pathological types, $>80 \%$ of TC is papillary thyroid carcinoma (PTC) (1). A total of $15-30 \%$ thyroid nodules evaluated using fine-needle aspiration (FNA) are not clearly benign or malignant (2). Genomic alterations have been fully interpreted by studies in recent years. The Cancer Genome Atlas (TCGA) Research Network performed the largest cohort study of PTC, and created a comprehensive multiplatform analysis (3). A previous study by Ye et al (4) drew the genetic landscape of benign thyroid nodules and thyroid cancer, and suggested that PTC and benign nodules have independent origins (4).

BRAF V600E is the most common mutation observed in PTC (3), and was recommended for the evaluation of thyroid nodules by the NCCN Guidelines of Thyroid Carcinoma (5). However, tumor tissue DNA sequencing has limitations. Tumors are heterogeneous, and the results of sequencing of a tumor section do not represent the whole tumor (6). Additionally, a surplus of tissues following FNA cytology is difficult to obtain, or the amount obtained may not be sufficient for sequencing to be performed. Circulating tumor DNA (ctDNA) is the cell-free fragment of DNA, which is released into the bloodstream by tumor cells, and the ctDNA load is associated with tumor staging and prognosis (7). Therefore, the detection of these known somatic gene mutations in tumors and the corresponding plasma has the potential to aid in the diagnosis of thyroid tumors. Previous studies have confirmed that there is controversy regarding ctDNA detection in patients with thyroid tumors. Although some studies have clarified the clinical significance of ctDNA (8-10), others have raised the issue that the analysis of ctDNA cannot improve the clinical management of patients with thyroid carcinoma, due to plasma ctDNA being undetectable $(11,12)$. 
In the present study, whole-exome sequencing (WES) was performed on tumor and matched normal tissues from 10 patients with PTC from Quanzhou, China, and a genomic alteration profile was created. The validation cohort, including 59 patients with PTC, was recruited to detect hotspot mutations in tumor DNA and cell-free DNA using QuantStudio ${ }^{\mathrm{TM}}$ 3D digital PCR. The accuracy of ctDNA detection was analyzed according to the sequencing results of the tumor DNA.

\section{Materials and methods}

Patients and samples. A total of 59 patients (Fig. 1) with PTC were recruited from Quanzhou First Hospital Affiliated to Fujian Medical University (Quanzhou, China) between August 2017 and June 2018. None of them had received therapeutic procedures such as chemotherapy. For 10 of the 59 patients, tumor and matched normal tissues from these patients were sampled during surgery, stored at $-80^{\circ} \mathrm{C}$ then subjected to WES (Table I). The matched normal tissues were collected which were at least $3 \mathrm{~cm}$ distance from cancer-foci. Tumor tissue and plasma were collected from all 59 patients, whose clinical characteristics are presented in Table II. The TMN Classification of Malignant Tumors (TNM) staging refers to AJCC Cancer Staging Manual Eighth Edition (13). All surgical specimens were pathologically diagnosed as PTC, and patients with other malignant tumors were excluded. Fresh tissues for WES were snap-frozen in liquid nitrogen immediately following surgical resection and subsequently stored at $-80^{\circ} \mathrm{C}$. Tumor tissues for PCR, from 49 patients, were formalin-fixed and paraffin embedded (FFPE) and collected from the pathology department following surgery. Tissues were fixed in $10 \%$ neutral formalin overnight at room temperature before embedding in paraffin. Prior to surgery, $10 \mathrm{ml}$ peripheral blood was collected in a Cell-Free DNA BCT blood collection tube (Streck, Inc.), and the plasma was separated immediately and subjected to $800 \mathrm{x}$ g centrifugation for $10 \mathrm{~min}$ at $4^{\circ} \mathrm{C}$ and a $2500 \mathrm{x} \mathrm{g}$ centrifugation for $10 \mathrm{~min}$ at $4^{\circ} \mathrm{C}$.

DNA extraction. Genomic DNA (gDNA) from fresh tissues was extracted using a Hipure Tissue DNA Mini kit (Magen). gDNA was extracted from FFPE tissues using a GeneRead DNA FFPE kit (Qiagen China Co., Ltd.) and plasma cfDNA was extracted using a HiPure Circulating DNA Midi kit (Pharmaceutical Product Development, Inc.), according to the manufacturers' protocols. The quantity and purity of gDNA were assessed using a Qubit ${ }^{\circledR} 3.0$ Fluorometer (Invitrogen; Thermo Fisher Scientific, Inc.) and a NanoDrop ND-1000 device (Thermo Fisher Scientific, Inc.).

WES. A total of $300 \mathrm{ng}$ of each gDNA sample, based on the Qubit quantification, were mechanically fragmented (duty factor $10 \%$; peak incident power $175 \mathrm{~W}$; cycles per burst 150 ; treatment time $150 \mathrm{sec}$; bath temperature $4-8^{\circ} \mathrm{C}$ ) on a Covaris E220 focused ultrasonicator (Covaris, Inc.). Sheared gDNA (200 ng) was used to perform the end repairs, and A-tailing and adapter ligation was performed with KAPA Hyper Prep kit (cat. no. KK8504; Kapa Biosystems, Inc.), according to the manufacturer's protocol. Libraries were then captured using Agilent SureSelect Human All Exon (version 6; Agilent Technologies, Inc.) probes, and subsequently amplified.
Libraries were sequenced using an Illumina HiSeq 2500 platform (Illumina, Inc.) on high output mode, and 2x150 cycles were performed using TruSeq SBS chemistry (version 3; Illumina, Inc.).

Bioinformatics analysis. Clean data were obtained from filtering out the adapter, low-quality reads and reads with a proportion $>10 \%$. Reads were aligned to the reference human genome (UCSC hg19; http://genome.ucsc.edu/) using the Burrows-Wheeler Aligner (version 0.7.12) (14). Local realignment, PCR duplicate marking, base-quality recalibration, and calculation of coverage metrics were performed using GATK v.3.2 (15) and Picard tools (http://broadinstitute.github. io/picard/).

Somatic single-nucleotide variants and InDels of tumors compared with matched normal tissues were annotated using MuTect software (version 1.1.4) (16). The mutations with variant allele frequencies $>1 \%$ were filtered and the variants in non-coding regions and synonymous variants were excluded from analysis. Gene ontology (GO; http://www.geneontology. org/) and Kyoto Encyclopedia of Genes and Genomes (KEGG; https://www.kegg.jp/) enrichment analyses were performed in order to investigate the biological importance of the somatic mutated genes using ClusterProfiler (17) in R software (version 3.5.1) (18).

Hotspot mutations detected by QuantStudio ${ }^{T M} 3 D$ digital PCR. BRAF V600E and NRAS p.Q61R were detected using QuantStudio ${ }^{\mathrm{TM}}$ 3D Digital PCR Master Mix v2 (cat. no. A26358) in a QuantStudio ${ }^{\mathrm{TM}}$ 3D digital PCR system (both Thermo Fisher Scientific, Inc.). The primer sequences of BRAF V600E were as follows: Forward, 5'-CTACTGTTTTCCTTT ACTTACTACACCTCAGA-3' and reverse, 5'-ATCCAGACA ACTGTTCAAACTGATG-3'. The primer sequences of NRAS p.Q61R were as follows: Forward, 5'-CACACCCCCAGGATT CTTACA-3' and reverse, 5'-TGTATTGGTCTCTCATGGCAC TGT-3'. A solution containing $20 \mu \mathrm{l}$ PCR reaction solution was prepared. The QuantStudio ${ }^{\mathrm{TM}}$ 3D Digital PCR $20 \mathrm{~K}$ Chip kit v2 (cat. no. A26316; Thermo Fisher Scientific, Inc.) was used to prepare the PCR reaction with $14.5 \mu \mathrm{l}$ sealed sample oil, which was then placed in the sample slot of the PreFlex PCR system for PCR amplification. Procedures were performed at $95^{\circ} \mathrm{C}$ for $10 \mathrm{~min} ; 98^{\circ} \mathrm{C}$ for $30 \mathrm{sec}$; and $60^{\circ} \mathrm{C}$ for $2 \mathrm{~min}$, for a total of 45 cycles. The amplified chip was placed in the chip analyzer to read the data, and the QuantStudio ${ }^{\mathrm{TM}}$ 3D Analysis Suite software was used to quantify the number of mutations in the samples from the collected data in the QuantStudio ${ }^{\mathrm{TM}}$ 3D Digital PCR System.

Statistical analysis. Statistical analysis was performed using SPSS software (version 19.0; IBM Corp.). Differences in distributions between the clinicopathological characteristics and molecular status were assessed using a $\chi^{2}$ or Fisher's exact test, as appropriate. Linear regression analysis was performed and graphical plots were generated using GraphPad Prism software (version 6.0; GraphPad Software, Inc.), OriginPro 8.1 (OriginLab, Corp.) and R. The OncoPrint function of ComplexHeatmap package (19) in $\mathrm{R}$ was used to visualize genomic alterations. $\mathrm{P}<0.05$ was considered to indicate a statistically significant difference. 
Table I. Clinical characteristics and whole-exome sequencing quality control of patients with papillary thyroid carcinoma.

\begin{tabular}{|c|c|c|c|c|c|c|c|c|}
\hline Case no. & $\begin{array}{l}\text { Age, } \\
\text { years }\end{array}$ & Sex & Multifocality & TNM stage & $\begin{array}{c}\text { Tumor sequencing } \\
\text { depth }(\mathrm{x})\end{array}$ & $\begin{array}{c}\text { Normal sequencing } \\
\text { depth }(\mathrm{x})\end{array}$ & $\begin{array}{c}\text { TMB } \\
\text { (mutations/Mb) }\end{array}$ & $\begin{array}{l}\text { BRAF } \\
\text { V600E }\end{array}$ \\
\hline 1 & 40 & Female & Yes & T1aN0M0 & 187.32 & 99.89 & 0.29 & - \\
\hline 2 & 29 & Female & No & T1aN0M0 & 179.27 & 98.48 & 0.35 & - \\
\hline 3 & 43 & Female & No & T1aN0M0 & 169.37 & 86.95 & 0.38 & - \\
\hline 4 & 65 & Male & Yes & T1aNOM0 & 82.39 & 56.64 & 0.29 & - \\
\hline 5 & 38 & Female & Yes & T1aN1aM0 & 67.98 & 31.87 & 0.16 & - \\
\hline 6 & 45 & Female & No & T1aN1aM0 & 159.76 & 39.63 & 1.35 & + \\
\hline 7 & 34 & Female & No & T1aN1aM0 & 174.48 & 47.25 & 1.06 & + \\
\hline 8 & 37 & Female & No & T1aN1aM0 & 81.04 & 46.23 & 0.32 & + \\
\hline 9 & 29 & Female & No & T1aN1aM0 & 133.27 & 37.27 & 1.07 & + \\
\hline 10 & 55 & Male & No & T1aN1aM0 & 150.61 & 79.92 & 0.38 & + \\
\hline
\end{tabular}

TNM, Tumor-Node-Metastasis; TMB, tumor mutation burden.

Table II. Clinical characteristics of the validation cohort, and association with BRAF V600E status in tumors.

\begin{tabular}{|c|c|c|c|c|}
\hline \multirow[b]{2}{*}{ Clinical characteristic } & \multirow[b]{2}{*}{ No. patients } & \multicolumn{2}{|c|}{ BRAF V600E in tumors } & \multirow[b]{2}{*}{ P-value } \\
\hline & & Positive $(n=26)$ & Negative $(n=33)$ & \\
\hline Age (median, range) & $41(22-65)$ & $39(22-64)$ & $41(22-65)$ & 0.283 \\
\hline \multicolumn{5}{|l|}{ Sex } \\
\hline Male (\%) & $17(28.81 \%)$ & 7 & 10 & 0.078 \\
\hline Female $(\%)$ & $42(71.19 \%)$ & 19 & 23 & \\
\hline Tumor size, cm; median (range) & $1.8(0.2-5.4)$ & $1.7(0.2-4.6)$ & $2.3(0.6-5.4)$ & 0.658 \\
\hline \multicolumn{5}{|l|}{ LNM } \\
\hline Positive (\%) & $20(33.90)$ & 15 & 5 & $0.001^{\mathrm{b}}$ \\
\hline Negative $(\%)$ & $39(66.10)$ & 11 & 28 & \\
\hline \multicolumn{5}{|l|}{ Capsule invasion } \\
\hline Positive (\%) & $20(33.90)$ & 11 & 9 & 0.226 \\
\hline Negative (\%) & $39(66.10)$ & 15 & 24 & \\
\hline \multicolumn{5}{|l|}{ Stage } \\
\hline $\mathrm{I}+\mathrm{II}(\%)$ & $38(64.41)$ & 12 & 26 & $0.009^{\mathrm{a}}$ \\
\hline III+IV (\%) & $21(35.59)$ & 14 & 7 & \\
\hline
\end{tabular}

${ }^{\mathrm{a}} \mathrm{P}<0.01 ;{ }^{\mathrm{b}} \mathrm{P}<0.001 . \mathrm{LNM}$, lymph node metastasis.

\section{Results}

Clinical characteristics and whole-exome sequencing data. The clinical characteristics of the WES cohort and all patients with PTC are presented in Tables I and II, respectively. The median patient age was 41 years (range, 22-65 years). Of the 59 patients with PTC, 42 were female, and the remaining 17 were male. Tumors of $20(33.90 \%)$ patients exhibited lymph node metastasis (LNM), and capsule invasion also occurred in $20(33.90 \%)$ tumors.

For the sequencing depth of 10 patients with PTC, WES achieved an average of $138.55 x$ coverage of tumor genomes and $62.41 \mathrm{x}$ coverage of the germline genomes (Table I). The average tumor mutation burden was 0.565 (range, 0.29-1.35)
mutations/Mb. PTC presents a low frequency of somatic alterations compared with other types of carcinoma (3), which may contribute to the good prognosis exhibited by PTC.

Somatic gene mutations in PTC as identified using WES. Fig. 2A presents the WES results of 10 patients with PTC, including the top 20 mutated genes, mutation type and frequency. BRAF V600E was the hotspot mutation in PTC and exhibited a 50.0\% frequency, which was comparable to a TCGA network study of PTCs (59.7\% mutated frequency of BRAF V600E) and to other previous studies $(3,20-22)$. The results also revealed that five tumors with the BRAF V600E mutation were all from patients with PTC who exhibited lymph node metastasis. Digital PCR was used to validate the BRAF V600E-positive tumors that were 


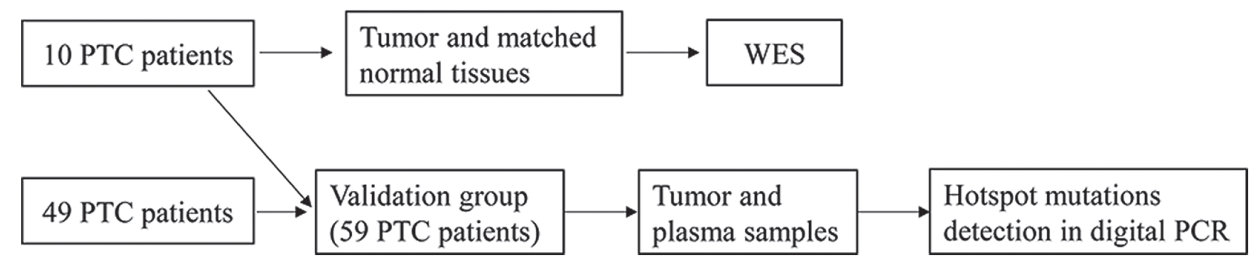

Figure 1. Schematic diagram of patients included in the current study. PTC, papillary thyroid carcinoma; WES, whole-exome sequencing.

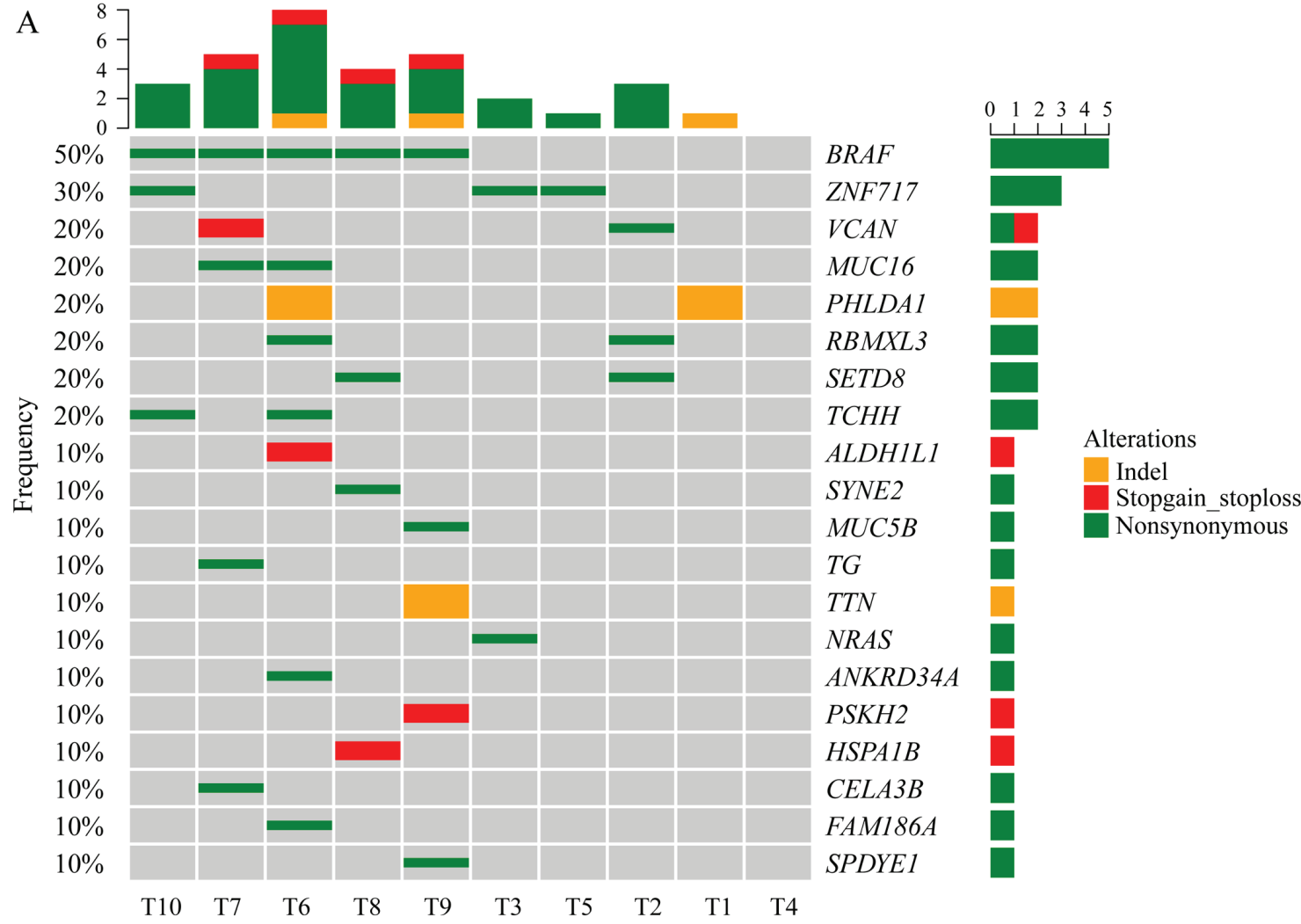

B

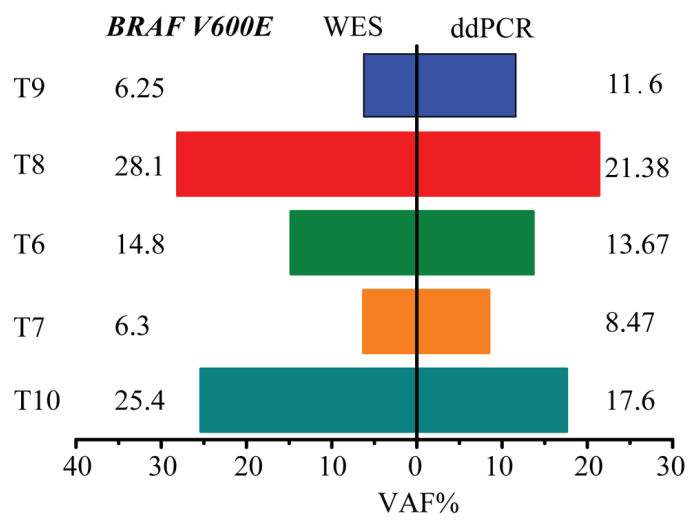

$\mathrm{C}$

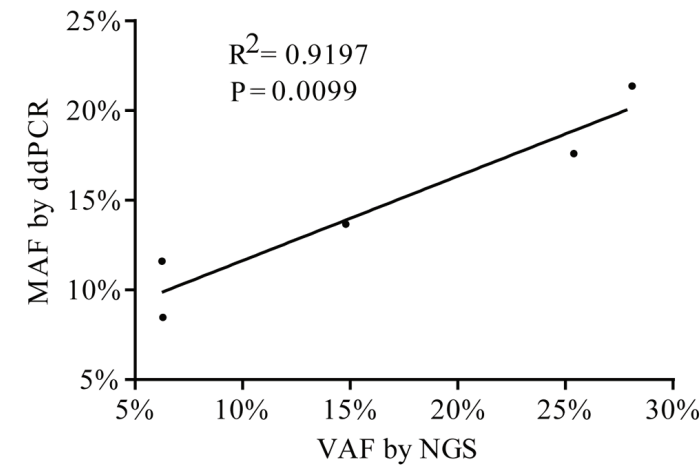

Figure 2. Somatic mutated genes in PTC. (A) Somatic mutation landsape of 10 patients with PTC using WES. (B) VAF of BRAF V600E in tumors detected by WES and digital PCR and (C) linear regression analysis. PTC, papillary thyroid carcinoma; WES, whole-exome sequencing; VAF, variation allele frequency.

detected using WES. The results of this analysis indicated a good degree of consistency in BRAF status (Fig. 2B) and variation allele frequency (VAF; Fig. 2C) between WES and digital PCR. VAF of BRAF V600E detected by WES had a positive linear correlation $\left(\mathrm{R}^{2}=0.9197 ; \mathrm{P}=0.0099\right)$ with digital PCR (Fig. 2C). A number of novel gene mutations were identified, including zinc finger protein (ZNF)717, pleckstrin homology like domain family A member 1 (PHLDA1), RBMX like 3 (RBMXL3), lysine methyltransferase 5A (SETD8) and trichohyalin (TCHH), along with reported genes, including BRAF, NRAS and mucin 16 (MUC16). 


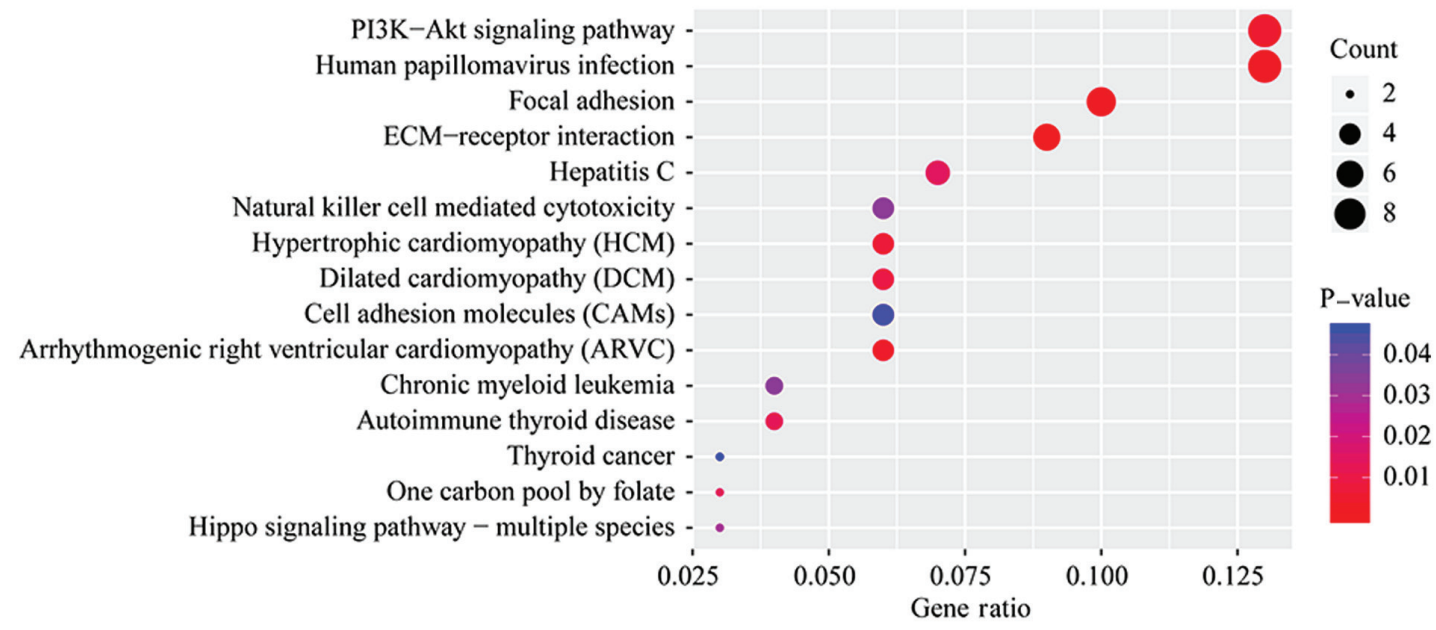

B

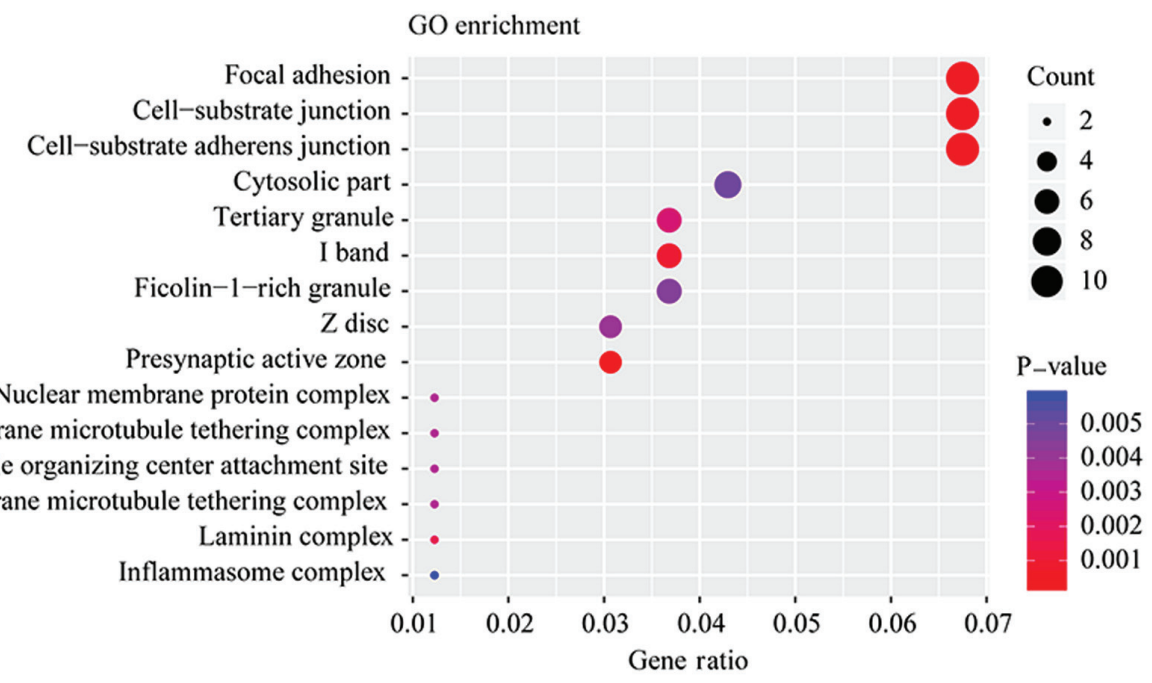

Figure 3. Pathway analysis of somatic mutated genes in PTC using (A) KEGG and (B) GO enrichment. PTC, papillary thyroid carcinoma; KEGG, Kyoto Encyclopedia of Genes and Genomes; GO, Gene ontology.

Functional enrichment analysis of mutated genes. To further determine the biological function of all mutated genes, KEGG and GO enrichment were performed. Fig. 3A and B presents the top 15 pathways and biological functions enriched by KEGG and GO analysis, respectively, according to their P-values. 'Focal adhesion' was a pathway that was significantly altered according to both the KEGG and GO enrichment analyses. Adhesion to the substrate via specific focal adhesion points has previously been considered an essential step in cell migration (23). In the present study, 11 mutated genes were enriched in three biological functions: 'Focal adhesion', 'cell-substrate junction' and 'cell-substrate adherens junction', including cadherin 2, heat shock family A member 1B, PTPRF interacting protein alpha 1 , spectrin repeat containing nuclear envelope protein 2, transcriptional adaptor 1, basigin, integrin subunit- $\alpha$ (ITGA) 4 , trio and f-actin binding protein, ZNF185, ITGA8 and NHS actin remodeling regulator.

Hotspot mutations detected by digital PCR in the tumor and plasma tissue of the validation cohort. The clinical significance of BRAF and RAS mutations has been previously studied (20), and it has been recommended that the diagnosis of thyroid nodules should occur using indeterminate FNA results according to the NCCN guidelines for thyroid carcinoma (5). To evaluate the accuracy of ctDNA detection compared with tumor tissues, BRAF and NRAS hotspot mutations were detected in the plasma and tumor tissues in the validation group using digital PCR. For the tumor tissues of the 59 patients with PTC, the BRAF V600E mutation frequency was $44.07 \%$ (Table II). Among the 26 patients with BRAF V600E tumors, BRAF V600E mutation was detected in the cell-free DNA of the plasma of 16 patients, while BRAF mutations in cell-free DNA were detected in 3 patients from the 33 with BRAF wild-type tumors. The sensitivity and specificity of BRAF V600E in cell-free DNA of patients with negative and positive results, detected by digital PCR, were 61.54 and 90.91\%, respectively (Table III).

As presented in Table II, BRAF V600E in patients' tumor tissues was significantly associated with advanced stage $(\mathrm{P}=0.009)$ and LNM $(\mathrm{P}=0.001)$. However, no significant association was observed with age, sex, tumor size or capsule invasion. The characteristics of the tumors (including stage, tumor size and LNM) were also investigated to determine whether these influenced the sensitivity of BRAF V600E in 
Table III. Sensitivity and specificity of BRAF V600E and NRAS Q61R detection in plasma in papillary thyroid carcinoma, according to the results gained from tumor tissue analysis.

\begin{tabular}{lcccc}
\hline & & \multicolumn{2}{c}{ Mutation in plasma } & \\
\cline { 3 - 3 } Tumor mutation & Negative & Positive & Sensitivity (\%) & Specificity (\%) \\
\hline BRAF V600E & 30 & 3 & 61.54 & 90.91 \\
Negative & 10 & 16 & & 98.18 \\
Positive & 54 & 1 & 50 & \\
NRAS p.Q61R & 2 & 2 & & \\
Negative & & & & \\
Positive & & & & \\
\hline
\end{tabular}

Table IV. Association between cell-free BRAF V600E status and tumor characteristics in 26 patients with BRAF V600E-positive tumors.

\begin{tabular}{lccr}
\hline & \multicolumn{2}{c}{ BRAF V600E in plasma } & \\
\cline { 2 - 3 } Characteristic & Negative & Positive & P-value \\
\hline Stage, no. patients & & & 0.422 \\
I+II & 6 & 6 & \\
III+IV & 4 & 10 & \\
LNM, no. patients & & & 1.000 \\
Negative & 4 & 7 & \\
$\quad$ Positive & 6 & 9 & \\
Tumor size, cm; & $1.55(0.2-3.6)$ & $1.7(0.4-4.6)$ & 0.766 \\
median (range) & & & \\
\hline
\end{tabular}

LNM, lymph node metastasis.

plasma. Table IV demonstrated that there was no significant correlation between the sensitivity of BRAF V600E in the plasma and clinical parameters, despite there being a marked trend.

NRAS p.Q61R was also detected in the tumor tissues and plasma in the validation cohort. NRAS p.Q61R was present in four tumors $(6.78 \%)$. The sensitivity of NRAS p.Q61R in ctDNA was $50.00 \%$ and the specificity was $98.18 \%$ (Table III). Low frequency of NRAS p.Q61R in tumors limits its independent application for liquid biopsy whereas combined detection of BRAF V600E and NRAS p.Q61R in plasma is feasible.

\section{Discussion}

Cellular pathology following FNA remains the most reliable method of distinguishing benign and malignant thyroid tumors prior to surgery (24). However, the features of $10-30 \%$ of thyroid nodules are still unable to be confirmed by FNA (24), which leads to these patients receiving unnecessary surgical treatment. For these patients, the detection of genetic mutations in their tumor tissues, via fine needle puncture, can be used to screen high-risk patients with thyroid cancer (25), and help guide further treatment.
In the present study, WES was used to investigate the genomic alteration profile of 10 patients with PTC from Quanzhou, China. All 59 patients with PTC formed the validation cohort to verify the results. BRAF V600E was the most frequent mutation in the WES cohort (5/10) and the mutation frequency was $44.07 \%$ in the validation cohort (26/59), which was consistent with previous studies $(26,27)$. BRAF gene mutations have been reported to be associated with tumor progression, sensitivity to iodine treatment and poor prognosis $(26,28,29)$. The results from the present study revealed that BRAF V600E was significantly associated with an advanced disease stage $(\mathrm{P}=0.009)$ and LNM $(\mathrm{P}=0.001)$. Additionally, consistency was observed in the identification of the BRAF genotype and VAF between WES and digital detection, which provided evidence for the reliability of WES. Currently, the BRAF status, based on surgical pathology specimens, is used by clinicians to evaluate patients at risk of recurrence (20).

Mutated genes, including ZNF717, PHLDA1, RBMXL3, SETD8 and TCHH, were also identified in the present study. These genes were novel and had not been reported in the TCGA study (3). For example, ZNF717 mutated in three samples, which encodes a Kruppel-associated box zinc-finger protein (30). Duan et al (31) reported that ZNF717 was a potential driver gene in hepatocellular carcinoma, as a tumor suppressor acting through the regulation of the interleukin-6/signal transducer and activator of transcription 3 pathway. Further evaluation of these genes should be performed in future studies, with validation of these mutated genes in a larger cohort, and determination of their pathogenic mechanisms.

Liquid biopsy is beneficial in diagnosis and treatment of cancers (32). ctDNA can be tested without accessing tumor tissues. Additionally, the short half-life of ctDNA and its wide dynamic range (33) may allow for the rapid assessment of therapeutic responses for early- and late-stage disease. Previous studies have indicated that the detection of ctDNA in plasma can aid in cancer diagnosis and can guide treatment course (34), including liver cancer (35), colorectal cancer (36) and melanoma (37). However, controversy exists regarding the detection of ctDNA in the plasma of patients with thyroid cancer. Cradic et al (38) and Zane et al (9) demonstrated that the mutation rate of BRAF in the plasma of patients with thyroid cancer was low, and that this was therefore not associated with thyroid cancer. However, a study performed by Kim et al (22), which included 72 patients with thyroid cancer, 
revealed that gene mutations in cell-free DNA were positive in three patients with LNM and lung metastasis, and this study concluded that there was a positive correlation between ctDNA and lung metastasis in patients with thyroid carcinoma.

In the present study, BRAF V600E and NRAS p.Q61R were analyzed in the tumor tissue and plasma of 59 patients with PTC in the validation cohort, and the accuracy of ctDNA detection was analyzed according to the results from the QuantStudio $^{\text {TM }}$ 3D PCR. The sensitivity of BRAF V600E and NRAS p.Q61R in ctDNA was 61.54 and 50.00\%, respectively, and the specificity was 90.91 and $98.18 \%$, respectively.

In the present study, for BRAF V600E, the ctDNA samples of $38.46 \%$ patients with positive BRAF V600E tumors were negative. In a study performed by Pupilli et al (10), the corresponding ctDNA was negative in 22 PTC patients whose tumors were BRAF V600E positive, using reverse transcription-PCR and digital PCR. In the aforementioned study, the release efficiency of DNA from the tumor to the peripheral blood was affected by a variety of factors, such as capsule invasion, size and tumor stage. ctDNA was not detected when its concentration was lower than the sensitivity limitation of QuantStudio ${ }^{\mathrm{TM}}$ 3D PCR. Additionally, there exists a high risk of degradation for cell-free DNA due to its short half-life (39). Few tumors in this aforementioned study were wild-type, and the sequencing results of the corresponding plasma were mutated, which may be accounted for by this. Tumors possess heterogeneity, and the sequencing results of a small section of tumor tissue cannot represent the entire tumor (6). Additionally, this potential false-sensitive result could be caused by the ultrahigh sensitivity of digital PCR.

In conclusion, the present study drew a genomic alteration profile of patients with PTC from southeast China, and provided evidence for the accuracy of ctDNA analysis in patients with PTC. ctDNA is an important tool for assisting in the diagnosis of thyroid nodules, predicting prognosis and tracking the progress of PTC when tumor tissue cannot be obtained.

\section{Acknowledgements}

Not applicable.

\section{Funding}

This work was supported by the Natural Science Foundation of Fujian Province (grant no. 2014J01435), Quanzhou Science and Technology Project (grant no. 2013Z58), and Projects of Special Development Funds in Zhangjiang National Independent Innovation Demonstration Zone, Shanghai (grant no. ZJ2017-ZD-012).

\section{Availability of data and materials}

The datasets used and/or analyzed during the current study are available from the corresponding author on reasonable request.

\section{Authors' contributions}

QP and HL wrote the manuscript; HL, ZS and SW contributed to the study design and manuscript revision; JZhan, ML and
CW collected samples and performed PCR; SW performed whole-exome sequencing; JZhao analyzed the whole-exome sequencing data; QP collected clinical data, analyzed the PCR data and developed the figures and tables. All authors read and approved the final manuscript.

\section{Ethics approval and consent to participate}

Ethical approval for the recruitment of human subjects was obtained from the Ethics Committee of Affiliated Quanzhou First Hospital of Fujian Medical University and was consistent with ethical guidelines provided by the Declaration of Helsinki (1975). Written informed consent was obtained from each patient.

\section{Patient consent for publication}

All individuals whose data were included in the current study provided informed consent for publication.

\section{Competing interests}

The authors declare that they have no competing interests.

\section{References}

1. Prescott JD and Zeiger MA: The RET oncogene in papillary thyroid carcinoma. Cancer 121: 2137-2146, 2015.

2. Alexander EK, Kennedy GC, Baloch ZW, Cibas ES, Chudova D, Diggans J, Friedman L, Kloos RT, LiVolsi VA, Mandel SJ, et al: Preoperative diagnosis of benign thyroid nodules with indeterminate cytology. N Engl J Med 367: 705-715, 2012.

3. Cancer Genome Atlas Research Network: Integrated genomic characterization of papillary thyroid carcinoma. Cell 159: 676-690, 2014.

4. Ye L, Zhou X, Huang F, Wang W, Qi Y, Xu H, Yang S, Shen L, Fei X, Xie J, et al: The genetic landscape of benign thyroid nodules revealed by whole exome and transcriptome sequencing. Nat Commun 8: 15533, 2017.

5. National Comprehensive Cancer Network (NCCN): NCCN Clinical Practice Guidelines in Oncology. Thyroid Carcinoma (Version 1.2019). https://www.nccn.org/professionals/physician_gls/default.aspx\#thyroid. Accessed March 28, 2019.

6. Burrell RA, McGranahan N, Bartek J and Swanton C: The causes and consequences of genetic heterogeneity in cancer evolution. Nature 501: 338-345, 2013.

7. Diaz LA Jr and Bardelli A: Liquid biopsies: Genotyping circulating tumor DNA. J Clin Oncol 32: 579-586, 2014.

8. Allin DM, Shaikh R, Carter P, Thway K, Sharabiani MTA, Gonzales-de-Castro D, O'Leary B, Garcia-Murillas I, Bhide S, Hubank M, et al: Circulating tumour DNA is a potential biomarker for disease progression and response to targeted therapy in advanced thyroid cancer. Eur J Cancer 103: 165-175, 2018.

9. Zane M, Agostini M, Enzo MV, Casal Ide E, Del Bianco P, Torresan F, Merante Boschin I, Pennelli G, Saccani A, Rubello D, et al: Circulating cell-free DNA, SLC5A8 and SLC26A4 hypermethylation, BRAF(V600E): A non-invasive tool panel for early detection of thyroid cancer. Biomed Pharmacother 67: 723-730, 2013.

10. Pupilli C, Pinzani P, Salvianti F, Fibbi B, Rossi M, Petrone L, Perigli G, De Feo ML, Vezzosi V, Pazzagli M, et al: Circulating BRAFV600E in the diagnosis and follow-up of differentiated papillary thyroid carcinoma. J Clin Endocrinol Metab 98: 3359-3365, 2013.

11. Condello V, Macerola E, Ugolini C, De Napoli L, Romei C, Materazzi G, Elisei R and Basolo F: Analysis of circulating tumor DNA does not improve the clinical management of patients with locally advanced and metastatic papillary thyroid carcinoma. Head Neck 40: 1752-1758, 2018.

12. Lupo M, Guttler R, Geck Z, Tonozzi TR, Kammesheidt A and Braunstein GD: Is measurement of circulating tumor DNA of diagnostic use in patients with thyroid nodules? Endocr Pract 24: 453-459, 2018. 
13. AJCC Cancer Staging Manual, 8th Edition. Springer, New York, NY, 2017.

14. Li H and Durbin R: Fast and accurate short read alignment with Burrows-Wheeler transform. Bioinformatics 25: 1754-1760, 2009.

15. McKenna A, Hanna M, Banks E, Sivachenko A, Cibulskis K, Kernytsky A, Garimella K, Altshuler D, Gabriel S, Daly M and DePristo MA: The Genome Analysis Toolkit: A MapReduce framework for analyzing next-generation DNA sequencing data. Genome Res 20: 1297-1303, 2010.

16. Cibulskis K, Lawrence MS, Carter SL, Sivachenko A, Jaffe D, Sougnez C, Gabriel S, Meyerson M, Lander ES and Getz G: Sensitive detection of somatic point mutations in impure and heterogeneous cancer samples. Nat Biotechnol 31: 213-219, 2013.

17. Yu G, Wang LG, Han Y and He QY: ClusterProfiler: An R package for comparing biological themes among gene clusters. OMICS 16: 284-287, 2012.

18. R Core Team: R: A language and environment for statistical computing. R Foundation for Statistical Computing, Vienna, Austria, 2018. https://www.R-project.org.

19. Gu Z, Eils R and Schlesner M: Complex heatmaps reveal patterns and correlations in multidimensional genomic data. Bioinformatics 32: 2847-2849, 2016.

20. Xing M, Alzahrani AS, Carson KA, Shong YK, Kim TY, Viola D, Elisei R, Bendlová B, Yip L, Mian C, et al: Association between BRAF V600E mutation and recurrence of papillary thyroid cancer. J Clin Oncol 33: 42-50, 2015.

21. Kim TH, Park YJ, Lim JA, Ahn HY, Lee EK, Lee YJ, Kim KW, Hahn SK, Youn YK, Kim KH, et al: The association of the BRAF(V600E) mutation with prognostic factors and poor clinical outcome in papillary thyroid cancer: A meta-analysis. Cancer 118: 1764-1773, 2012.

22. Kim BH, Kim IJ, Lee BJ, Lee JC, Kim IS, Kim SJ, Kim WJ, Jeon YK, Kim SS and Kim YK: Detection of plasma BRAF(V600) mutation is associated with lung metastasis in papillary thyroid carcinomas. Yonsei Med J 56: 634-640, 2015.

23. Paluch EK, Aspalter IM and Sixt M: Focal adhesion-independent cell migration. Annu Rev Cell Dev Biol 32: 469-490, 2016.

24. Cibas ES and Ali SZ: The 2017 Bethesda system for reporting thyroid cytopathology. Thyroid 27: 1341-1346, 2017.

25. Haugen BR, Alexander EK, Bible KC, Doherty GM, Mandel SJ, Nikiforov YE, Pacini F, Randolph GW, Sawka AM, Schlumberger M, et al: 2015 American thyroid association management guidelines for adult patients with thyroid nodules and differentiated thyroid cancer: The American thyroid association guidelines task force on thyroid nodules and differentiated thyroid cancer. Thyroid 26: 1-133, 2016.

26. Xing M, Alzahrani AS, Carson KA, Viola D, Elisei R, Bendlova B, Yip L, Mian C, Vianello F, Tuttle RM, et al: Association between BRAF V600E mutation and mortality in patients with papillary thyroid cancer. JAMA 309: 1493-1501, 2013.

27. Lin KL ZX and Dai XX: Detection of BRAF gene mutations in patients with high risk papillary thyroid carcinoma before the operation. Chin J Oncol 33: 130-131, 2011.
28. Prescott JD, Sadow PM, Hodin RA, Le LP, Gaz RD, Randolph GW Stephen AE, Parangi S, Daniels GH and Lubitz CC: BRAF ${ }^{\mathrm{V} 600 \mathrm{E}}$ status adds incremental value to current risk classification systems in predicting papillary thyroid carcinoma recurrence. Surgery 152: 984-990, 2012.

29. Riesco-Eizaguirre G, Gutiérrez-Martínez P, García-Cabezas MA, Nistal M and Santisteban P: The oncogene BRAF V600E is associated with a high risk of recurrence and less differentiated papillary thyroid carcinoma due to the impairment of Na+/Itargeting to the membrane. Endocr Relat Cancer 13: 257-269, 2006.

30. Muzny DM, Scherer SE, Kaul R, Wang J, Yu J, Sudbrak R, Buhay CJ, Chen R, Cree A, Ding Y, et al: The DNA sequence, annotation and analysis of human chromosome 3 . Nature 440 : 1194-1198, 2006.

31. Duan M, Hao J, Cui S, Worthley DL, Zhang S, Wang Z, Shi J, Liu L, Wang X, Ke A, et al: Diverse modes of clonal evolution in HBV-related hepatocellular carcinoma revealed by single-cell genome sequencing. Cell Res 28: 359-373, 2018.

32. Fiala $C$ and Diamandis EP: Utility of circulating tumor DNA in cancer diagnostics with emphasis on early detection. BMC Med 16: 166, 2018.

33. Mengual L, Lozano JJ, Ingelmo-Torres M, Gazquez C, Ribal MJ and Alcaraz A: Using microRNA profiling in urine samples to develop a non-invasive test for bladder cancer. Int J Cancer 133: 2631-2641, 2013.

34. Bettegowda C, Sausen M, Leary RJ, Kinde I, Wang Y, Agrawal N, Bartlett BR, Wang H, Luber B, Alani RM, et al: Detection of circulating tumor DNA in early- and late-stage human malignancies. Sci Transl Med 6: 224ra24, 2014.

35. Ye Q, Ling S, Zheng S and Xu X: Liquid biopsy in hepatocellular carcinoma: Circulating tumor cells and circulating tumor DNA. Mol Cancer 18: 114, 2019.

36. Garlan F, Laurent-Puig P, Sefrioui D, Siauve N, Didelot A, Sarafan-Vasseur N, Michel P, Perkins G, Mulot C, Blons H, et al: Early evaluation of circulating tumor DNA as marker of therapeutic efficacy in metastatic colorectal cancer patients (PLACOL study). Clin Cancer Res 23: 5416-5425, 2017.

37. Tan L, Sandhu S, Lee RJ, Li J, Callahan J, Ftouni S, Dhomen N, Middlehurst P, Wallace A, Raleigh J, et al: Prediction and monitoring of relapse in stage III melanoma using circulating tumor DNA. Ann Oncol 30: 804-814, 2019.

38. Cradic KW, Milosevic D, Rosenberg AM, Erickson LA, McIver B and Grebe SK: Mutant BRAF(T1799A) can be detected in the blood of papillary thyroid carcinoma patients and correlates with disease status. J Clin Endocrinol Metab 94: 5001-5009, 2009.

39. Khier S and Lohan L: Kinetics of circulating cell-free DNA for biomedical applications: Critical appraisal of the literature. Future Sci OA 4: FSO295, 2018.

This work is licensed under a Creative Commons Attribution-NonCommercial-NoDerivatives 4.0 International (CC BY-NC-ND 4.0) License. 